\title{
Impacto de las redes sociales y sus descontroladas distribuciones masivas.
}

\section{Impact of social networks and their uncontrolled mass distributions.}

\author{
Pablo Homero Velasteguí. ${ }^{1}$
}

\section{Resumen.}

En este artículo le doy una clara explicación sobre el cambio de los adolescentes por las nuevas tecnologías le damos una clara explicación de lo negativo y positivo de las redes sociales en los adolescentes y el abuso de las tecnología.

También se dice que la nueva tecnología en la actualidad a afectado simultáneamente a las niñez y jóvenes porque hoy en día en las noticias se ve muchas cosas que suceden en todo el mundo hay abuso de identidad abuso sexual ya que las personas pedófila utilizan la redes sociales como una herramienta de conocer niñas e adolecentes para satisfacer sus necesidades en la actualidad hay muchas tristeza ya que padre de familia Allan a sus hijas muerta violada que han sido víctima de los pedófilo y que sus formas de enredo son las amenaza por medio de las redes sociales hay personas que amenazan a las niñas.

En los último año se ha producido una revolución tecnológica con la creación de internet por parte del departamento de defensa de estado unidos, se ha creado nuevo aparato tecnológico como computadora personales teléfonos integrales y Tablet generando un cambio global y que son muy bueno para la comunicación ya que con estos dispositivo nos podemos comunicar con las personas querida que se encuentra muy lejos en otros países.

Son millones los individuos en todo el mundo, especialmente adolecente y jóvenes ,que se han dejado seducir por las nuevas tecnología e internet, incorporándolos en su vida cotidiana, en sus comunicaciones y en sus vínculo familiar. Sin embargo, la aparición de diferentes aplicaciones ha estado ligado al desarrollo de conducta adictiva, en algunos individuos con diferente conducta, los jóvenes de hoy en día son adictivo al internet.

Palabras claves: Adolescencia internet, identidad, redes sociales, comunicación, supervisión adulta.

\footnotetext{
${ }^{1}$ Ciencia digital, Ambato, Ecuador, luisefrainvelastegui@ cienciadigital.org
} 


\section{Abstract.}

In this article I give a clear explanation about the change of adolescents by the new technology we give a clear explanation of the negative and positive of social networks in adolescents and the abuse of technology.

It is also said that the new technology is currently affected simultaneously by children and young people because today in the news you see many things happening around the world there is abuse of identity sexual abuse since pedophiles use social networks as a tool to meet girls and adolescents to meet their needs today there are many sadness as Allan father of his daughters dead children who have been victimized by pedophiles and that their entanglements are threatening them through networks There are people who threaten girls.

In the last year there has been a technological revolution with the creation of the internet by the United States Department of Defense, a new technological device has been created, such as personal computers, integral phones and tablets, generating a global change and which are very good for communication. because with these devices we can communicate with loved ones who are far away in other countries.

There are millions of individuals all over the world, especially adolescents and young people, who have been seduced by new technology and the Internet, incorporating them into their daily lives, their communications and their family ties. However, the appearance of different applications has been linked to the development of addictive behavior, in some individuals with different behavior, young people today are addictive to the internet.

Keywords: Adolescence internet, identity, social networks, communication, adult supervision.

\section{Introducción}

En la actualidad hay muchos casos que suceden en los países y en el mundo entero casos como de violación acoso sexual abuso físico y verbal, pero hay muchos pedófilo que por medio del internet las redes sociales las cogen como una herramienta para hacer sus fechoría o simplemente para satisfacer sus necesidades conociendo muchachas de todo lado y comienzan a enamorarla y lo peor es que hay situaciones que son amenazada, también utilizan el internet para robar dinero o identidades y actualmente la adolescencia utilizan este medio de herramienta para satisfacer sus necesidades viendo pornografía.

\section{Criterio de los autores}

\section{Tipo de redes sociales}

Según soledad, 2013. DA, 2011. Proposed, 2010 Research, 2012. S, 2014 Mosso, 2008.definen que en la actualidad hay una gran variedad de redes sociales pero también hay ciertas redes que han contado con un mayor índice de éxito que el resto de redes si nos concentramos al nivel nacional y dentro de España las redes sociales más conocida y utilizada son:

Facebook: es una de las redes más utilizada nacional y mundial es una red que es sumamente gratuita y fue creada por Mark Zuckerberg. Esta red es la más utilizable por 
los jóvenes para poder conocer personas hacerse amigo con demás personas lejana pero los autores han mencionado que por medio de esta herramienta ávido mucho acoso como es 1 groo Ming, ciberbulling, sexting, y lo más que sucede adictivo al internet.

Twitter: una de las redes poco utilizable para los jóvenes esta red se creó en marzo del 2016 fue creada por Jack Dorsey esta red permite 1 usuario de la misma expresarse a través de publicaciones y comentario de cita longitud.

Tiene alrededor de 140 caracteres llamado "tweets". Estos caracteres contiene lo que son: video, fotos, enlace o ubicación sin traspasar el límite citado.

Por medio de esta red el usuario puede seguir o tiene la oportunidad de ser seguido por otros usuarios quiere decir que está atento a tus publicaciones o comentario quiere decir que si tu publicas algún tipo de video e imagen las personas que te siguen pueden ver tus publicaciones y también si los demás usuarios publican y tú lo sigues te llegan una notificación que el usuario " $\mathrm{X}$ " a publicado y tienes privilegio a comentar o darle un toque o likes.

Tuenti: esta red es más utilizada por los jóvenes se trata de una red española dirigida a la población joven. Se denomina como una plataforma de red social para la comunicación fue creada en el 2006 por Zaryn Dentzel.

Se trata de una red e plataforma similar a Facebook que se creó para la juventud menos de 25 años esta plataforma permite al usuario compartir publicaciones video foto e imagen enlace contenido de trabajo etc. Esta plataforma te da un privilegio que tu desea quien puede ver tus publicaciones o quien no es similar al Facebook

Linkedin: es un sitio web fue creado por Reid Hoffman en el 2002 fue destinado para los negocios.

Proporcionando a las empresas una manera rápida y cómoda de poner en contacto a las personas activa en la búsqueda de empleo de empresa y las empresas que ofertan puesto de trabajo quiere decir que es una plataforma para buscar trabajo y escoger empleado por medio de esta plataforma envían en curriculum virtual para obtener algún empleo en algunas empresas que está en esta plataforma.

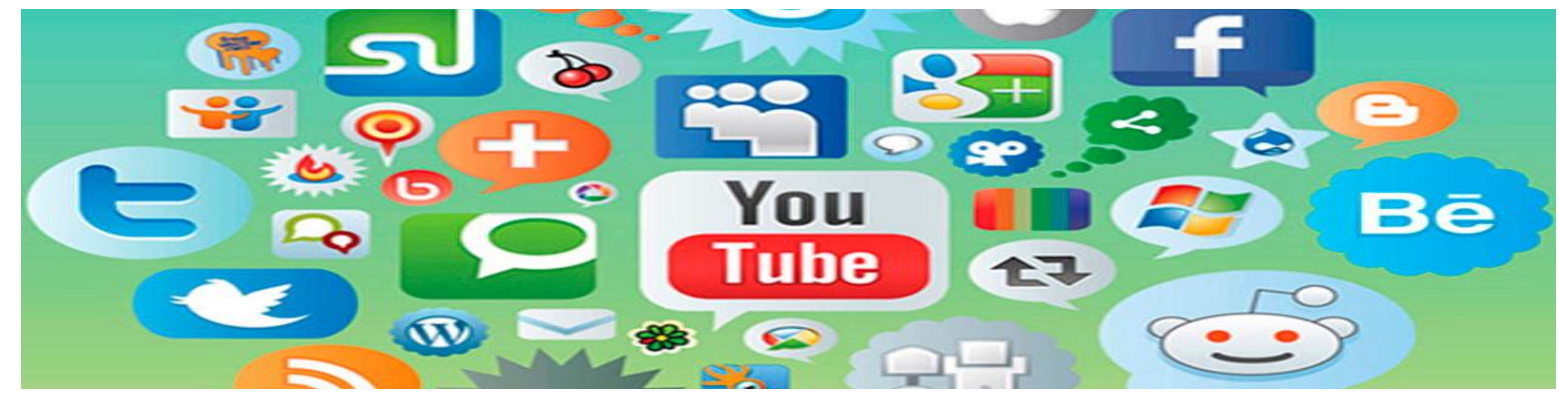

Autor: los creadores de las redes sociales ante mencionado

Fuente: tipo de redes sociales

Estadística del uso de las redes sociales 


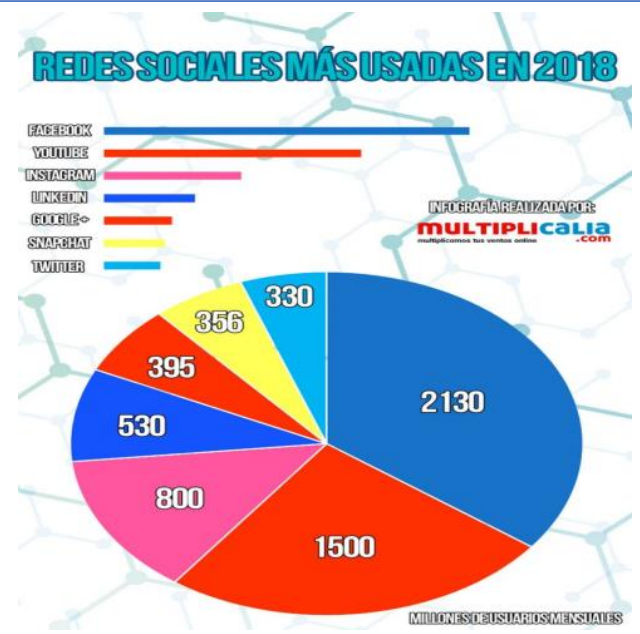

Estas son los tipos de redes sociales que hay en la actualidad hay le muestro la estadística de utilización de las redes en todo el mundo.

Estadística de los países que más utilizan las redes sociales.

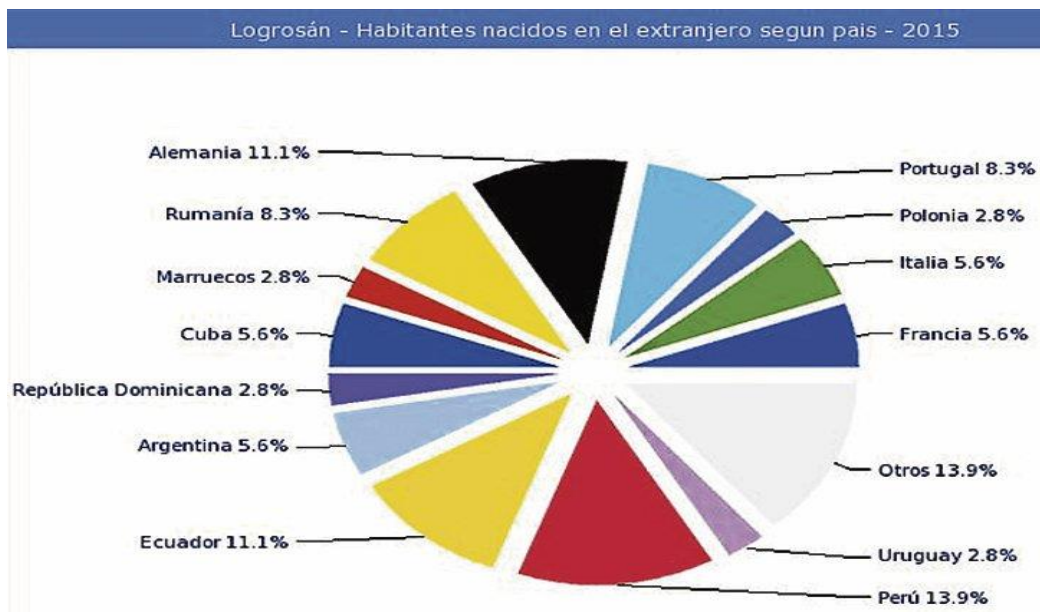

Estas son la estadística de las redes sociales que se utilizan en todos los países el porcentaje de la utilización de las redes.

\section{Criterio personal}

Como se aclaró en las opiniones de los autores dan a entender que hay algunos tipos de redes algunas sirven para conocer gente de otros lados publicar tus experiencias en el lugar que estas también tiene el privilegio de comentar alguna publicación del demás usuario y compartir para que las demás personas que tienes de contacto vean tus publicaciones.

Hay redes que sirven para buscar trabajo y publicar empleo para que las ciudadanías se informen cuando es el sueldo y a qué cargo van, pero si tienen algún tipo de estudio por medio de esta red informática se puede enviar el curriculum digital.

Pero hay un gran problema en la actualidad que hay muchas personas que cogen las redes sociales como herramienta para hacer maldades hoy en día los pedófilo son los que tienen 
más amigas mujeres para poder atacar o se crean cuenta falta para obtener algunas informaciones de los demás usuario mujeres.

Por medio de este documento le quiero dar un consejo a los padres de familia y a los jóvenes no se dejen creer de las personas que les escriben por las redes sociales que nadie sabe el mal de la olla solo la cuchara y a los padres sean más cuidadoso con sus hijos revísenle con quien escriben pregúntenle quien le hace daño sean buen padres.

\section{Criterio de los autores}

\section{Aspecto negativo del uso de las redes sociales}

Según M, 2011,2012. Valkenburg PM, 2011. Pichón Riviere, 1985. Watzlawick, 1993. (Müller KW, 2014,2016. Las opiniones de los autores que últimamente ha sido una alta cifra de la utilización de las redes sociales en la adolescencia y jóvenes. Estas redes sociales tienen una fuerte atracción que ejercen en el grupo tan vulnerable por su proceso neuropsicobiologico y sociales.

Por eso es muy necesario revisar y informarse cuáles son las causa de la utilización del internet quiere decir que estas tipo de redes sociales produce un nuevo estilo de comunicación quiere decir que si un usuario tiene mucho tiempo en el Facebook no aprende a vivir con los demás en persona solo se expresa por medio del internet en la vida cotidiana ya no quiere vivir solo se expresan por las redes sociales.

El abuso de las redes sociales ha mostrado una asociación con depresión, síndrome de déficit atencional con hiperactividad, insomnio, disminución de horas total de sueño, disminución del rendimiento académico, repotencia y abandono escolar.

Los Adolescentes que juegan de forma excesiva tienen menos capacidad de procesar el feedback frente a las decisiones, no considerándolo a la hora de tomarlas. Se ha visto, además, fallas en los procesos de aprendizaje En relación al desarrollo de personalidad, se ha registrado que, a mayor gravedad de la personalidad, mayor es el riesgo de adicción.

En relación a los aspectos negativos del uso de redes sociales en particular y de objetos tecnológicos en general, sabemos que la exposición a violencia en los medios de comunicación, internet, videojuegos y redes sociales, aumenta las interacciones agresivas en niños y adolescentes en encuadres sociales estructurados. Se produce, además, un alto grado de exposición deseada y no deseada a pornografía en usuarios de internet y existe una mayor exposición en individuos vulnerables (con tendencia a la delincuencia, con depresión y/o que han sido victimizados). Se genera una baja capacidad de lectura facial y de habilidades sociales directas.

Estas son las preguntas de las personas depredadora más conocido como los pedófilo

Amistad. El abusador se hace pasar por otra joven y se gana la confianza de la víctima, seduciéndola y obteniendo así sus datos personales (¿Qué edad tienes?, ¿Con quién vives?, ¿Cuál es tu dirección?, ¿Qué hacen tus padres?, ¿En qué colegio estás?). 
Engaño. El abusador finge estar enamorado de la víctima para conseguir que se desnude y realice actos de naturaleza sexual frente a la webcam, o le envíe fotografías de igual tipo.

Chantaje. El abusador manipula a la víctima amenazándola con que va a hacer público el material sexual, si no continúa enviándoselo. Las repercusiones del grooming en la víctima están asociadas a depresión, baja autoestima, desconfianza, cambios de humores repentinos y bruscos, bajo rendimiento académico, aislamiento, alteraciones del sueño y de la alimentación, ideas e intentos de suicidio.

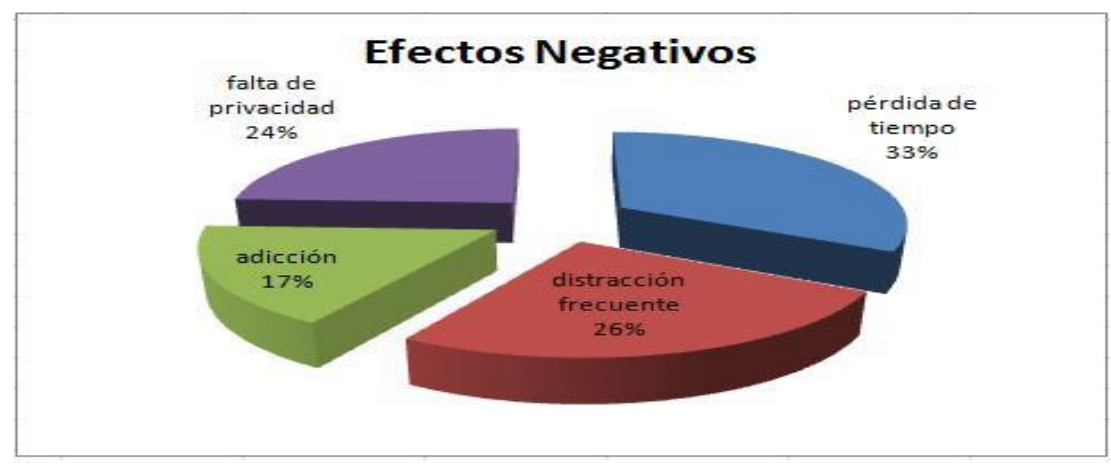

Autor: Alejandra de mayo del 2015

Fuente: Aspecto negativo de las redes sociales

Estas son las estadísticas por la cual es necesario saber los aspectos negativos de las redes sociales.

\section{Criterio personal}

En mi opinión que debemos de saber muy bien de las personas que estamos escribiéndolo por la cual hay mucho sucedo que en realidad son muy penoso las persona que engañan a las muchachas se hacen pasar como mujeres o como sus mejore amigo(a) para poder agradarle y hace cometer su acto estas personas son muy peligrosas ya que no quieren tener una amistad lo que quieren es hacer daño.

\section{Criterio de los autores}

\section{Aspecto positivo de las redes sociales}

Según Yao YW, 2014. Dalbudak E, 2010. KD, 2005. Wolak J, 2007. Bergquist T, 2009. Bennett N, 2014. Abufhele \& Arab, 2008. Definen que los aspectos positivos de las redes sociales y los más utilizados son que permite la comunicación con personas que viven lejos de nosotros, además permiten compartir imágenes, videos, música con las personas que deseemos con la ventaja que son gratuitos, y lo único que se necesita para ello es tener una cuenta en cualquiera de estas redes al igual que la persona que va a recibir la información.

Las redes sociales pueden ser positivas o negativas de acuerdo al uso que se les dé, pues que si se les usa de una manera negativa ósea para molestar o haces peores cosas con los demás obviamente son perjudiciales, pero si lo usan para hablar o comunicarse con 
personas o amigos que necesitas comunicarse ya sea por relaciones personales o de trabajo puede transformarse en una herramienta muy positiva.

\section{Aspectos positivos de las redes sociales}

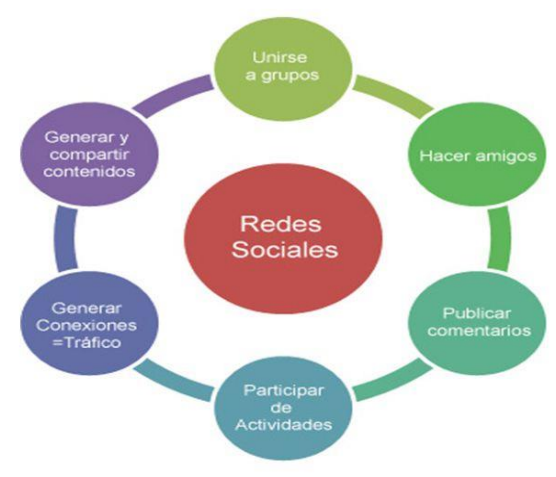

Autor: Efraín García

Fuente: Aspecto positivo de las redes sociales

Estas son la clara explicación por la cual es positivo tener una cuenta en algunas redes sociales

\section{Criterio personal}

En mi criterio del tema estoy muy bien primero porque los creadores de Facebook al creado una manera de rehabilitación para los adictos al internet para los obsesivo.

Es muy importante tener una cuenta por que por medio de esta red social por medio de esta plataforma nos podemos comunicar con las demás familias que están lejos y podemos conocer gente de otros lugares y lo más importante también nos podemos informar sobre la naturaleza los animales la flora y fauna son aspecto muy importante.

\section{Conclusiones}

En todo este tema que hemos visto le doy una clara conclusión de todos en general se dice que el impacto de las redes sociales más influencia han tenido los jóvenes quienes son lo que utilizan las redes para conocer personas lejanas cerca y para tener una amistad con ciertas personas las redes sociales más influente son: Facebook, Twitter, WhatsApp son las redes sociales más influente en todo el mundo.

$>$ Debido a estas redes se han notado muchas problemáticas asesinado secuestro extorsiones y violaciones ya que hay enfermos sexuales que utilizan las redes sociales como una herramienta para conocer muchachas e invitarla a salir para poder satisfacer sus necesidades las violan y las asesinan. Estos tipos de persona se hacen para por un Facebook falso las engañan primeramente se les hacen los amigos les pregunta cómo están donde eres con quien vives por dónde vives etc. 
$>$ Las redes sociales son muy buenas porque hace podemos conocer mucha gente y tener una comunicación con los seres querido de otros países también nos permite comunicarnos con los animales la naturaleza en la actualidad hay un gran índice de adicción la adicción no solo es consumir drogas algunas sustancia hay jóvenes que se vuelven adicto al internet a las redes sociales ya que no pueden vivir sin un teléfono una semana parecen que el mundo se le va las redes sociales es una plataforma adictiva que los jóvenes no pueden detener hay jóvenes y adolecente que ingresan a su cuenta más de 3 a 6 veces al día y si tienen un aparato electrónico se quedan hasta de madrugada conversando con " $X$ " personas estas tipo de redes lo desanima a la juventud de vivir el mundo exterior tienen desanimo emocional para poder conversar con una persona cercana no quieren hacer sus deberes no van al colegio.

Es muy importante tener una clara información sobre las redes sociales para poder manejar la adicción.

\section{Referencias Bibliográficas}

(s.f.).

Abufhele, M., \& Arab, E. (01 de septiembre de 2008). Obtenido de Abufhele, Marcela; Arab, Elías. el fenómeno del "Bullying". Caracterización del problema y sus estrategias de intervención". Revista Chilena de Psiquiatría y Neurología de la Infancia y Adolescencia, volumen 19-no1-septiembre 2008, issn-0718-3798.

Bennett N, O. W. (25 de septiembre de 2014). Obtenido de Bennett N, O’Donohue W. The Construct of Grooming in Child Sexual Abuse: Conceptual and Measurement Issues. J Child Sex Abus. 2014 Sep 25. [Epub ahead of print] PubMed PMID: 25258355.

Bergquist T, G. C. (23 de septiembre de 2009). Obtenido de Bergquist T, Gehl C, Mandrekar J, Lepore S, Hanna S, Osten A, et al. The effect of internet-based cognitive rehabilitation in persons with memory impairments after severe traumatic brain injury. Brain Inj. 2009 Sep;23(10):790-9. doi: 10.1080/0269905090319

DA, c. (junio de 2011). Obtenido de Problematic internet usage in US college students: a pilot study. BMC Med. 2011 Jun 22;9:77. doi: 10.1186/1741-7015-9-77. PubMed PMID: 21696582; PubMed Central PMCID: PMC3141542.

Dalbudak E, E. C. (2015). Obtenido de Dalbudak E, Evren C, Aldemir S, Evren B. The severity of Internet addiction risk and its relationship with the severity of borderline personality features,childhood traumas, dissociative experiences, depression and anxiety symptoms among Turkish universit 
KD, B. (19 de febrero de 2005). Obtenido de Browne KD, Hamilton-Giachritsis C. The influence of violent media on children and adolescents:a public-health approach. Lancet. 2005 Feb19-25;365(9460):702-10. Review. PubMed PMID: 15721477.

M, I. (2011,2012). Obtenido de , Kim T, Bukobza G. Adolescents' over-use of the cyber world- Internet addiction or identity exploration? J Adolesc. 2012 Apr;35(2):417-24. doi: 10.1016/j.adolescence.2011.07.015. Epub 2011 Jul 3 PubMed PMID: 21803411.

Mosso, P. (2008). Obtenido de Mosso, Penjerek. Yo me cuido ¿sol@?, salud y prevención en la adolescencia. Editorial Maipue, Argentina, 2008.

Müller KW, J. M. (2014,2016). Obtenido de Müller KW, Janikian M, Dreier M, Wölfling $\mathrm{K}$, Beutel ME, Tzavara C, et al. Regular gaming behavior and internet gaming disorder in European adolescents: results from a cross-national representative survey of prevalence, predictors, and psychopathological c

Pichón Riviere, E. (1985). Obtenido de Pichón Riviere, Enrique. El Proceso Grupal: Del Psicoanálisis a la Psicología Social. Editorial Nueva Visión, Buenos Aires, 1985.El Proceso Grupal: Del Psicoanálisis a la Psicología Social. Editorial Nueva Visión, Buenos Aires, 1985.

Proposed, R. J. (agosto de 2010). Obtenido de Rey J. Proposed changes to the psychiatric classification: towards DSM5. Australas Psychiatry. 2010 Aug;18(4):309-13.

Research, C. (2012). Obtenido de Criteria Research Santiago, Chile, 2012. http://www.criteria.cl/category/estudios-de-tendencias/.

S, L. (junio de 2014). Obtenido de . Annual research review: Harms experienced by child users of online and mobile technologies: the nature, prevalence and management of sexual and aggressive risks in the digital age. J Child Psychol Psychiatry. 2014 Jun;55(6):635-54

soledad, r. (2013). Obtenido de LGuan SS, Subrahmanyam K. Youth Internet use: risks and opportunities. Curr Opin Psychiatry. 2009 Jul;22(4):351-6. doi: 10.1097/YCO.0b013e3283 2bd7e0. Review. PubMed PMID: 19387347.

Valkenburg PM, P. J. (febrero de 2011). Obtenido de Valkenburg PM, Peter J. Online communication among adolescents: an integrated model of its attraction, opportunities, and risks. J Adolesc Health. 2011 Feb;48(2):121-7. doi: 10.1016/j.jadohealth.2010.08.020. Epub 2010 Dec 13. Review. PubMed PMID: 21257109

Watzlawick, P. (1993). Obtenido de Watzlawick, Paul. Teoría de la Comunicación Humana. Editorial Herder, 1993. 
Wolak J, M. K. (19 de febrero de 2007). Obtenido de Wolak J, Mitchell K, Finkelhor D. Unwanted and wanted exposure to online pornography in a national sample of youth Internet users. Pediatrics. 2007Feb;119(2):247-57. PubMed PMID: 17272613.

Yao YW, C. P. (2014). Obtenido de Yao YW, Chen PR, Chen C, Wang LJ, Zhang JT, Xue G, et al. Failure to utilize feedback causes decision-making deficits among excessive Internet gamers. Psychiatry Res. 2014 Nov 30;219(3):583-8. doi:10.1016/j. psychres.2014.06.033. Epub 2014 Jun 28. PubMed 


\section{Para citar el artículo indexado.}

Velasteguí P. (2018) Impacto de las redes sociales y sus descontroladas distribuciones masivas. Revista electrónica Explorador Digital 2(2), 47-57. Recuperado desde:

http://cienciadigital.org/revistacienciadigital2/index.php/exploradordigital/article/view/332/7 44

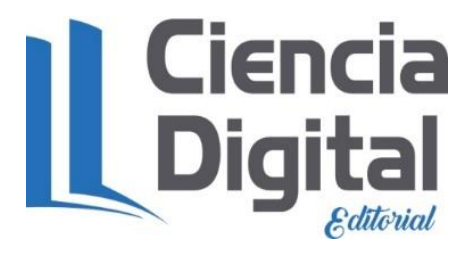

El artículo que se publica es de exclusiva responsabilidad de los autores y no necesariamente reflejan el pensamiento de la Revista Explorador Digital.

El articulo queda en propiedad de la revista y, por tanto, su publicación parcial y/o total en otro medio tiene que ser autorizado por el director o editor de la Revista Explorador Digital.
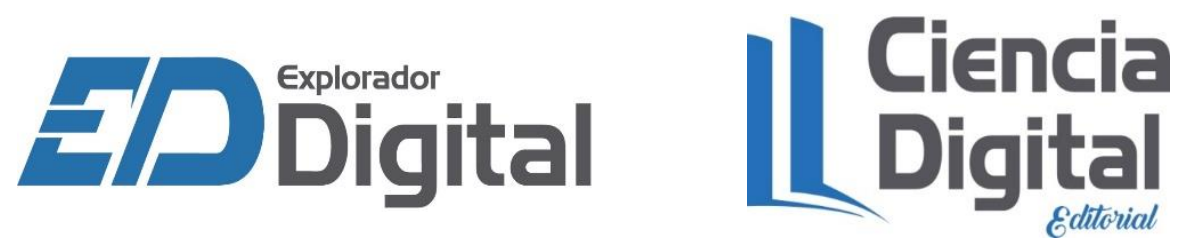